\title{
Molecular confirmation of CHARGE syndrome from umbilical cord blood stem cells from a deceased newborn and identification of a new mutation in the exon 29 of the CHD7 gene
}

\author{
Nélida Montano ${ }^{1^{*}}$, Andrea Quadrelli $^{1}$, Aubrey Milunsky ${ }^{2}$, Alicia Vaglio ${ }^{1}$, Roberto Quadrelli ${ }^{1}$ \\ ${ }^{1}$ Instituto de Genética Médica, Hospital Italiano, Montevideo, Uruguay; *Corresponding Author: rquadr@dedicado.net.uy \\ ${ }^{2}$ Center for Human Genetics, Boston University School of Medicine, Boston, USA
}

Received 15 September 2011; revised 20 October 2011; accepted 30 October 2011

\begin{abstract}
CHARGE syndrome (Coloboma of the eye, Heart defects, Atresia of the choanae, Retardation of growth and/or development, Genital and/or urinary abnormalities, and Ear abnormalities) is an autosomal dominant disorder characterized by a specific and a recognizable pattern of anomalies. De novo mutations in the CHD7 gene are the major cause of CHARGE syndrome. Here, we present a family who sought genetic counseling because of a newborn with dysmorphic features suggesting CHARGE syndrome. The baby died three months later. Afterwards, a molecular genetic testing for sequence analysis of the CHD7 coding region was performed with DNA extracted from umbilical cord blood stem cells confirming the diagnosis of CHARGE syndrome. Although the diagnosis is first suspected clinically, in the newborn case presented here, we illustrate the importance of the molecular testing to confirm the diagnosis, and to enable precise genetic counseling. Also, even though cord blood has been stored in private banks for more than ten years, there is as yet no routine clinical application of autologous (self-donation) hematopoietic stem cells from cord blood. Now, we illustrate for the first time the usefulness of umbilical cord blood stem cells for diagnosis and genetic counseling in a case that involve a dead propositus.
\end{abstract}

Keywords: CHARGE Syndrome; CHD7 Gene; Genetic Counseling; Umbilical Cord Blood Stem Cells

\section{INTRODUCTION}

CHARGE syndrome is an autosomal dominant disorder with a prevalence of about one in 10,000 [1]. The acronym CHARGE is based on the cardinal features identified when the syndrome was delineated: coloboma, heart malformation, choanal atresia, retardation of growth and/or development, genital anomalies and ear anomalies [2]. The life expectancy of patients with CHARGE syndrome varies widely, with individuals living anywhere from five days to at least 46 years $[1,3,4]$. Mutations in the $\mathrm{CHD7}$ gene were identified as causative for CHARGE syndrome in approximately $2 / 3$ of patients with a clinical diagnosis of CHARGE syndrome [5,6]. Of the $\mathrm{CHD7}$ mutations reported thus far, approximately $72 \%$ are nonsense or frameshift, $13 \%$ are splice site, and $10 \%$ are missense [4]. Recurrent mutations are rare, and clear genotype-phenotype correlations have not been recognized [3,4,7]. CHD7 is located in $8 \mathrm{q} 12.1$; it is $188 \mathrm{~kb}$ in size, consists of 37 coding exons and one non-coding exon, and codes for a 2997 amino-acid protein. The function of $C H D 7$ protein is still largely unknown [2]. Pathogeny of the CHARGE remains puzzling: $\mathrm{CHD7}$ is a regulatory element that potentially affects a large number of development pathways, explaining the pleiotropic nature of its phenotypic spectrum [2]. Originally, the diagnosis of CHARGE syndrome included the identification of four to six cardinal signs, one being either choanal atresia or a coloboma [2]. Afterward, new diagnostic criteria were developed, expanding the original picture to encompass brainstem anomalies and visceral malformations [8] or focusing on the colobomachoanal atresia-abnormal semicircular canals triad, and giving a formal definition for partial and atypical CHARGE syndromes [2,9]. A combination of coloboma, choanal atresia and abnormal semicircular canals (3C) is highly predictive of the presence of a CHD7 mutation [2]. Prenatal diagnosis of CHARGE syndrome has been reported at least twice, although more than 200 newborns and infants with CHARGE syndrome have been described $[2,10]$. 
Here, we present a family who sought genetic counseling because of a newborn with dysmorphic features suggesting CHARGE syndrome. The baby died three months later. A molecular genetic testing for sequence analysis of the CHD7 coding region was performed with DNA extracted from umbilical cord blood stem cells. Umbilical cord blood stem cells can be viewed as the stem cells source of choice for clinical and non-clinical research applications [11]. Between other reasons, umbilical cord blood stem cells can be considered as one of the most abundant sources of non-embryonic stem cells, the collection is non-invasive, can be stored and cryopreserved in cord blood banks for later uses and occupy and intermediate age stage between the embryonic stem cells and the adult stem cells, which lead to a higher proliferating potential $[11,12]$. At this time, we illustrate for the first time the usefulness of umbilical cord blood stem cells for cases that involve a dead propositus.

\section{MATERIALS AND METHODS}

This case is about a family who sought genetic counseling because of a newborn with dysmorphic features including a square-shaped face with narrow bifrontal diameter, broad nasal bridge, short webbed neck, ocular asimmetry with left palpebral ptosis and ear and hand anomalies. The baby also showed chorioretineal coloboma, choanal atresia, anterior located anus and congenital heart disease. Visual and auditory evoked potentials were defined as pathological. The presenting features suggested the diagnosis of CHARGE syndrome. We recommended a sequence analysis of the CHD7 coding region but it was not performed at that time. The baby died three months later. Afterwards, the family decided to perform the molecular genetic testing. DNA was extracted from umbilical cord blood stem cells stored in the New England Cryogenic Center (NECC) and the sequence analysis of the CHD7 coding region was performed in the Center for Human Genetics (Boston University School of Medicine). Analysis of blood samples from patient's parents was also recommended.

\section{Molecular Genetic Testing}

DNA from the baby patient was extracted from umbilical cord blood stem cells. Briefly, fluorescence labeled PCR primers in exon flanking regions were used to amplify and sequence coding exons 2 - 38 in the CHD7 gene in both directions. Exon numbering is based on the NCBI reference NM_017780.2 sequence (2010). This service is performed pursuant to an agreement with Roche Molecular Systems, Inc. This test was developed and its performance characteristics determined by the Center for Human Genetics as required by the CLIA'88 regulations.
DNA from patient's parents was performed on peripheral blood lymphocytes. Briefly, PCR amplification and automated fluorescence sequencing was performed on exon 29 and its intervening sequence and exon 38 and its intervening sequence of the $\mathrm{CHD} 7$ gene.

Also, paternity and maternity testing was performed. Briefly, PCR analysis was used to test the DNA samples for 16 polymorphic markers using the ABI Identifier kit. Amplified DNA fragments were resolved by capillary gel electrophoresis. A paternity index was calculated based on marker frequencies in the population that are published or as derived from our accumulated database (Center for Human Genetics, Boston University School of Medicine).

\section{RESULTS}

Paternity and maternity was confirmed. DNA samples from the umbilical cord blood stem cells were tested at 16 unlinked DNA marker loci and the baby shares alleles with patient's alleged parents at all 16 tested loci. Analysis of the umbilical cord blood stem cells revealed that the baby was heterozygous for the L1898X alteration in exon 29 of the CHD7 gene. This alteration has not been previously reported in the literature; however, given that it is a truncating alteration, it likely confirms a diagnosis of CHARGE syndrome. This patient was also found to be heterozygous for the G2914R alteration in exon 38 of the CHD7. No additional mutations were found in the remaining 35 exons of $C H D 7$. Given the above result, analysis by multiplex ligation-dependant probe amplification (MLPA) to detect whole-exon or whole-gene deletions/duplications in CHD7 was not performed. These analyses detect approximately $65 \%$ of mutations in individuals with clinically diagnosed CHARGE syndrome.

On the other hand, patient's father was found to be negative for the L1898X alteration in exon 29 of the CHD7 gene, previously identified in this patient's son. Patient's mother was also found to be negative for the L1898X alteration in exon 29 of the CHD7 gene but heterozygous for the G2914R alteration in exon 38 of the CHD7 gene. The certainty of the molecular test result exceeds $99 \%$.

Hence, patient's parents were found to be negative for the L1898X mutation, which was interpreted as responsible for the clinical condition of CHARGE syndrome in the newborn. The mutation G2914R, found both in the mother's patient and the child, was considered to be a variant without pathological significance.

\section{DISCUSSION}

CHARGE syndrome is a clinical diagnosis; the clinical scores have shown their robustness, as almost all patients with CHD7 mutation are in accord with them 
[2]. Although the diagnosis is first suspected clinically, in the newborn case presented here, we illustrate the importance of the molecular testing of the $\mathrm{CHD} 7$ gene to confirm the diagnosis, and to enable precise genetic counseling. The molecular testing allowed the identification of a new mutation in exon 29 of the CHD7 gene from umbilical cord blood stem cells. Published mutation in CHD7 are scattered throughout the gene, and do not show preferential domain aggregation nor hot spot; mutations were shown to be dominantly transmitted in some families, and proven recurrence of a mutation in two sibs confirmed the possibility of germinal mosaicism [2]. Most of the mutations are truncating mutations that are likely to result in haploinsufficiency and most of the affected individuals have de novo mutations [7]. In the case presented here, the results suggest that the CHARGE syndrome of the newborn correspond to a de novo mutation with low risk of recurrence in future pregnancies of the couple. In fact, almost all cases of CHARGE syndrome are sporadic, although a small number of cases of familial CHARGE syndrome and parent-to-child transmission of CHD7 mutations have been reported [4]. As a result of the established diagnosis in this case, with low risk of recurrence, the couple decided to undergo a new pregnancy. The pregnancy outcome was an unaffected boy.

Here, we also illustrate for the first time the usefulness of umbilical cord blood stem cells for cases that involve a dead propositus. Privately run cord blood banks store cord blood for donors' own use and keep this for a certain period of time, for a fee that the parents pay to the company. As indicated before, it is possible that the parents regard this service as a sort of "biological life insurance policy" for their children; however, a scientific rationale and an indication for the use of such services are thus far lacking [13]. Allogenic umbilical cord blood stem cells (obtained from healthy donors, rather than from the patient to be treated), have been in routine use worldwide for more than ten years in the treatment of hematopoietic diseases [13,14]. On the other hand, autologous stem cells from cord blood have poor prospects for use in regenerative medicine, because they have to be cryopreserved until use [13]. In fact, there is as yet no routine clinical application of autologous hematopoietic stem cells from cord blood (self-donation of blood), even though cord blood has been stored in private banks for more than ten years [13]. Now, we demonstrate the value of umbilical cord blood stem cells for diagnosis and genetic counseling in a case that involve a dead propositus. This by itself may not be surprising but may encourage storing of cord blood not only for future stem cell-based therapies but to identify significant polymorphisms and mutations that may aid families after death of fetuses or other situations where tissues may not be available.

\section{REFERENCES}

[1] Issekutz, K.A., Graham, J.M. Jr., Prasad, C., Smith, I.M. and Blake, K.D. (2005) An epidemiological analysis of CHARGE syndrome: Preliminary results from a Canadian study. American Journal of Medical Genetics Part A, 133, 309-317. doi:10.1002/ajmg.a.30560

[2] Sanlaville, D. and Verloes, A. (2007) CHARGE syndrome: An update. European Journal of Medical Genetics, 15, 389-399.

[3] Jongmans, M.C., Admiraal, R.J., Van Der Donk, K.P., Vissers, L.E., et al. (2006) CHARGE syndrome: The phenotypic spectrum of mutations in the CHD7 gene. Journal of Medical Genetics, 43, 306-314. doi:10.1136/jmg.2005.036061

[4] Zentner, G.E., Layman, W.S., Martin, D.M. and Scacheri, P.C. (2010) Molecular and phenotypic aspects of CHD7 mutation in CHARGE syndrome. American Journal of Medical Genetics Part A, 152, 674-686. doi:10.1002/ajmg.a.33323

[5] Vissers, L.E., Van Ravenswaaij, C.M., Admiraal, R., et al. (2004) Mutations in a new member of the chromodomain gene family cause CHARGE syndrome. Nature Genetics, 36, 955-957. doi:10.1038/ng1407

[6] Johnson, D.S., Morrison, N., Grant, L., et al. (2006) Confirmation of $\mathrm{CHD7}$ as a cause of CHARGE association identified by mapping a balanced chromosome translocation in affected monozygotic twins. Journal of Medical Genetics, 43, 280-284. doi:10.1136/jmg.2005.032946

[7] Lalani, S.R., Safiullah, A.M., Fernbach, S.D., Harutyunyan, K.G., et al. (2006) Spectrum of CHD7 mutations in 110 individuals with CHARGE syndrome and genotype-phenotype correlation. American Journal of Human Genetics, 78, 303-314. doi:10.1086/500273

[8] Blake, K.D., Davenport, S.L., Hall, B.D., et al. (1998) CHARGE association: An update and review for the primary pediatrician. Clinical Pediatrics, 37, 159-173. doi:10.1177/000992289803700302

[9] Verloes, A. (2005) Updated diagnostic criteria for CHARGE syndrome: A proposal. American Journal of Medical Genetics Part A, 133, 306-308. doi:10.1002/ajmg.a.30559

[10] Sanlaville, D., Etchevers, H.C., Gonzales, M., Martinovic, J., Clément-Ziza, M., et al. (2006) Phenotypic spectrum of CHARGE syndrome in fetuses with $\mathrm{CHD7}$ truncating mutations correlates with expression during human development. Journal of Medical Genetics, 43, 211-217. doi:10.1136/jmg.2005.036160

[11] Ali, H. and Bahbahani, H. (2010) Umbilical cord blood stem cells-Potential therapeutic tool for neural injuries and disorders. Acta Neurobiologiae Experimentalis, 70, 316-324.

[12] Watt, S. and Contreras, M. (2005) Stem cell medicine: Umbilical cord blood and its stem cell potential. Seminars in Fetal and Neonatal Medicine, 10, 209-220. doi:10.1016/j.siny.2005.02.001

[13] Reimann, V., Creutzig, U. and Kögler, G. (2009) Stem 
cells derived from cord blood in transplantation and regenerative medicine. Deutsches Aerzteblatt international, 106, 831-836.

[14] Verneris, M.R., Brunstein, C.G., Barker, J., Macmillan, M.L., Defor, T., McKenna, D.H., et al. (2009) Relapse risk after umbilical cord blood transplantation: enhanced graft versus leukemia effect in recipients of tow units. Blood, 114, 4293-4299.

doi:10.1182/blood-2009-05-220525 